\title{
Parkinson's Disease Diagnosis and Severity Assessment Using Ground Reaction Forces and Neural Networks
}

\author{
Srivardhini Veeraragavan ${ }^{1 *}$, Alpha Agape Gopalai ${ }^{1 *}$, Darwin Gouwanda ${ }^{1}$ and \\ Siti Anom Ahmad ${ }^{2}$
}

${ }^{1}$ Advanced Engineering Platform, School of Engineering, Monash University Malaysia, Subang Jaya, Malaysia, ${ }^{2}$ Malaysian Research Institute on Ageing, Universiti Putra Malaysia, Selangor, Malaysia

\section{OPEN ACCESS}

Edited by:

Luca Mesin,

Politecnico di Torino, Italy

Reviewed by:

Antonio Brunetti,

Politecnico di Bari, Italy

Alessandro Marco De Nunzio,

Lunex University, Luxembourg

*Correspondence:

Srivardhini Veeraragavan srivardhini.veera@gmail.com

Alpha Agape Gopalai

alpha.agape@monash.edu

Specialty section:

This article was submitted to

Computational Physiology and Medicine,

a section of the journal

Frontiers in Physiology

Received: 20 August 2020

Accepted: 09 October 2020

Published: 09 November 2020

Citation:

Veeraragavan S, Gopalai AA,

Gouwanda D and Ahmad SA (2020)

Parkinson's Disease Diagnosis

and Severity Assessment Using

Ground Reaction Forces and Neural

Networks. Front. Physiol. 11:587057.

doi: 10.3389/fphys.2020.587057
Gait analysis plays a key role in the diagnosis of Parkinson's Disease (PD), as patients generally exhibit abnormal gait patterns compared to healthy controls. Current diagnosis and severity assessment procedures entail manual visual examinations of motor tasks, speech, and handwriting, among numerous other tests, which can vary between clinicians based on their expertise and visual observation of gait tasks. Automating gait differentiation procedure can serve as a useful tool in early diagnosis and severity assessment of PD and limits the data collection to solely walking gait. In this research, a holistic, non-intrusive method is proposed to diagnose and assess PD severity in its early and moderate stages by using only Vertical Ground Reaction Force (VGRF). From the VGRF data, gait features are extracted and selected to use as training features for the Artificial Neural Network (ANN) model to diagnose PD using cross validation. If the diagnosis is positive, another ANN model will predict their Hoehn and Yahr (H\&Y) score to assess their PD severity using the same VGRF data. PD Diagnosis is achieved with a high accuracy of $97.4 \%$ using simple network architecture. Additionally, the results indicate a better performance compared to other complex machine learning models that have been researched previously. Severity Assessment is also performed on the H\&Y scale with $87.1 \%$ accuracy. The results of this study show that it is plausible to use only VGRF data in diagnosing and assessing early stage Parkinson's Disease, helping patients manage the symptoms earlier and giving them a better quality of life.

Keywords: gait analysis, artificial neural \{network (ANN)\}, Parkinson's Disease, machine learning, SMOTE

\section{INTRODUCTION}

Parkinson's Disease (PD) is a highly prevalent neuro-degenerative disease that affects more than 10 million people worldwide. While PD usually occurs in adults aged 50 and above, there have been cases of young onsets of this disease, where individuals as young as 18 years old have been diagnosed with PD (Parkinson's Foundation, 2019). There are five progression stages in PD, where treatment in the early stages (Stages 1 and 2) slows down the onset of the disease, allowing patients to experience a better quality of life (Parkinson's Foundation, 2019). However, there is no specific test that exists to diagnose PD, and patients will have to rely on a neurologist for a diagnosis.

Neurologists typically base their diagnosis on several factors such as the patients' medical history, signs and symptoms exhibited, and a neurological and physical examination. Although there 
are existing scans which may help support neurologists' in verifying their diagnosis, it is the exhibited symptoms and neurological examination that carries the most weight in the diagnosis. This makes detection of PD in the early stages difficult as the exhibited symptoms are relatively mild and may require several visits to the neurologist before it can be confirmed (Parkinson's Foundation, 2019). These procedures can be taxing emotionally, financial and in terms of time for both patient and caregiver (Parkinson's Foundation, 2019).

Common symptoms observed by individuals suffering from PD include postural instability, tremor, slowness in movement and other forms of gait (Parkinson's Foundation, 2019) due to the deterioration of neurons in the brain. These symptoms start mildly and only escalate with the progression of the disease. Previous studies have investigated the potential of assessing changes in patterns of alteration in gait to aid the diagnosis and quantification of PD (Koozekanani et al., 1987; Salarian et al., 2004; Schlachetzki et al., 2017). These alterations were measured using non-intrusive wearable motion sensors which allow observation of natural day-to-day movements. These movements offer better insight into their individualized gait characteristics. The use of Force-Resistive Sensors (FRS) at the sole of the feet to measure gait events has been studied in the past (Goldberger et al., 2000), and also studies of FRS coupled with gyroscopes and accelerometers (Tadano et al., 2013; Bhosale et al., 2016). Given the significant advancements made in the miniaturization and processing speeds of these sensors, there is great potential in using wearable sensors for early diagnosis of PD.

A common conclusion from past studies of both diagnosis and severity assessment research is that a consistently higher gait cycle duration is observed in PD patients (Koozekanani et al., 1987; Salarian et al., 2004; Tadano et al., 2013; Bhosale et al., 2016; Schlachetzki et al., 2017). Recognizing this, we seek to investigate the possibility of using only wearable sensors to identify PD in its early stages and estimate PD severity. As a proof of concept, we use data previously reported in Goldberger et al. (2000). A key measurement in this dataset is the VGRF measured from the FRSs in the insoles of the feet. Many studies in the past have used this parameter to investigate and quantify gait variability of PD patients (Manap et al., 2011; Abdulhay et al., 2018). However, to the best of our knowledge, no study has explicitly researched on the sole use of gait features for severity assessment, as research in the area of severity assessment primarily focuses on the use of features extracted from speech data (Salarian et al., 2004; Benmalek et al., 2015; Schlachetzki et al., 2017; Grover et al., 2018; Nilashi et al., 2018), in addition to VGRF data. A successful implementation will enable early seamless diagnosis and assessment of PD using only VGRF data.

\section{EXPERIMENTAL SETUP}

\section{Data Description}

The database (Goldberger et al., 2000) consists of 93 idiopathic PD patients (58 male and 35 female, Age $=66.3 \pm 9.5$ years, Height of $1.67 \pm 0.084 \mathrm{~m}$ and an Weight of $72.4 \pm 11.83 \mathrm{~kg}$ ) and 73 healthy control subjects (40 male and 33 female, Age $=63.7 \pm 8.64$ years, Height of $1.68 \pm 0.085 \mathrm{~m}$ and Weight $=72.8 \pm 12.26 \mathrm{~kg}$ ).

The database includes the vertical ground reaction force (VGRF) records of subjects as they walked at their usual,

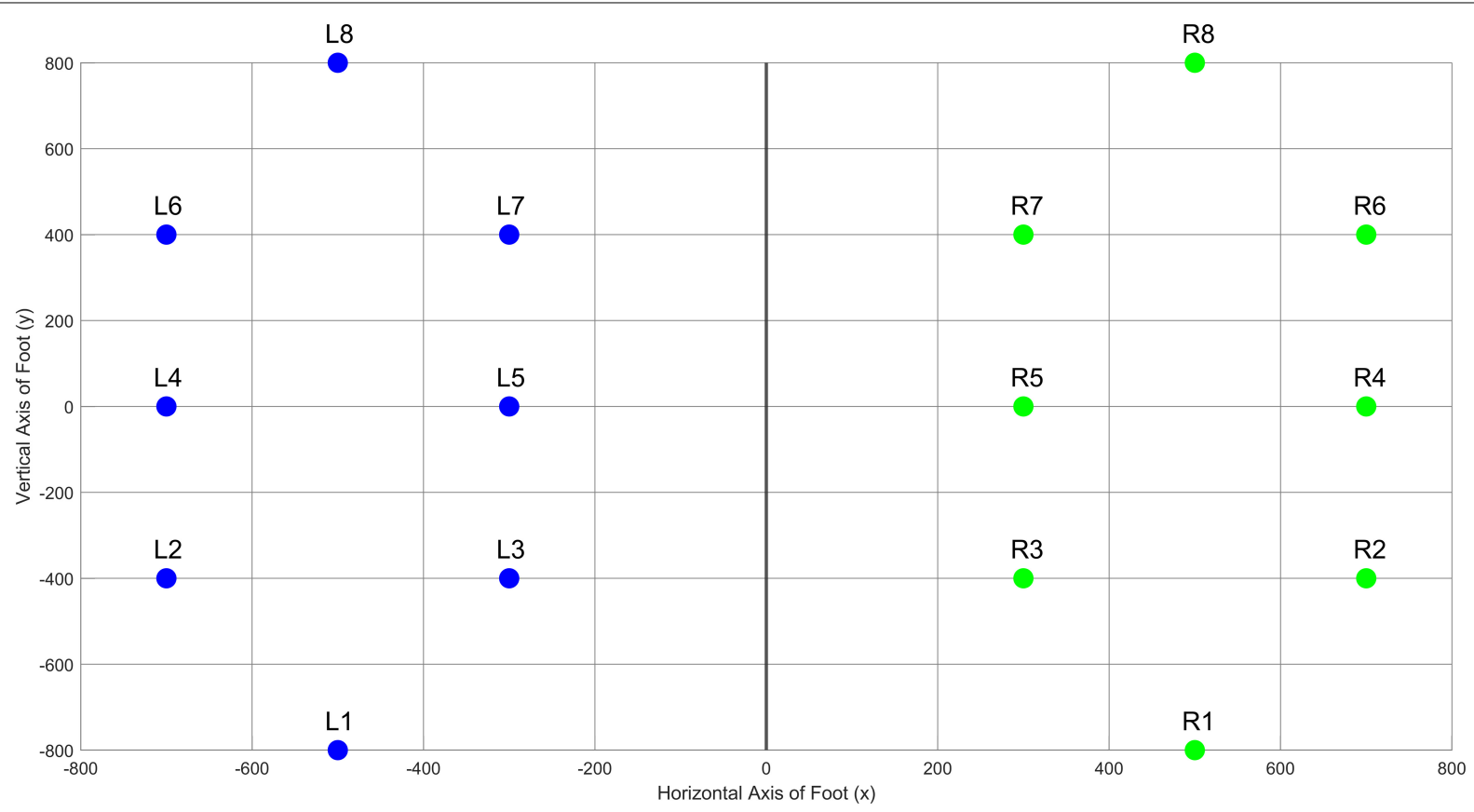

FIGURE 1 | Positioning of the sensors on an arbitrary relative coordinate system on the foot insole. 
self-selected pace for approximately $2 \mathrm{~min}$ on level ground. Underneath each foot were 8 sensors (Ultraflex Computer Dyno Graphy, Infotronics Inc.) that measure force (in Newtons) as a function of time. The 8 sensors are arranged on the soles of the feet as follows- 3 sensors each are placed along the inner and outer longitudinal arch, and a sensor each on base of the foot and the heel bone. The approximate coordinates of the sensor locations inside the insole are illustrated in Figure 1, whereby the $\mathrm{x}$ and $\mathrm{y}$ axes reflect an arbitrary coordinate system (Goldberger et al., 2000) to scale the sensor positions, with the origin in the center between both feet and the person is facing the positive side of the $y$ axis. This arbitrary coordinate system is relative to the positions of the sensors, thereby making the sensors inside the insole remain at the same relative coordinate during walking, but the feet are no longer parallel to each other (Goldberger et al., 2000). The output of each of these 16 sensors has been digitized and recorded at 100 samples per second, and the records also include two signals that reflect the sum of the 8 sensor outputs for each foot.
The database also includes qualitative measures of disease severity, including the Hoehn \& Yahr (H\&Y) staging for subjects suffering from PD. Clinicians use the H\&Y score to quantify the level of disability in patients (Parkinson's Foundation, 2019). A higher $\mathrm{H} \& \mathrm{Y}$ score corresponds to higher disease progression, and thus gait impairment associated with reduced mobility is more prevalent. The H\&Y score is a gross assessment of the level of disability through staging, and ranges from stages 0 to 5 , where 0 implies no signs of disease and 5 corresponds to a subject being fully impaired or bedridden. All the PD patients are of H\&Y stage between 2 and 3 (Average $=2.26 \pm 0.34$ ). This implies that the patients in this database are of an early to moderate onset of PD (Goldberger et al., 2000).

\section{Signal Processing and Feature Extraction}

All VRGF data were first filtered through a median filter to smoothen the signal and remove outliers (Salarian et al., 2004).

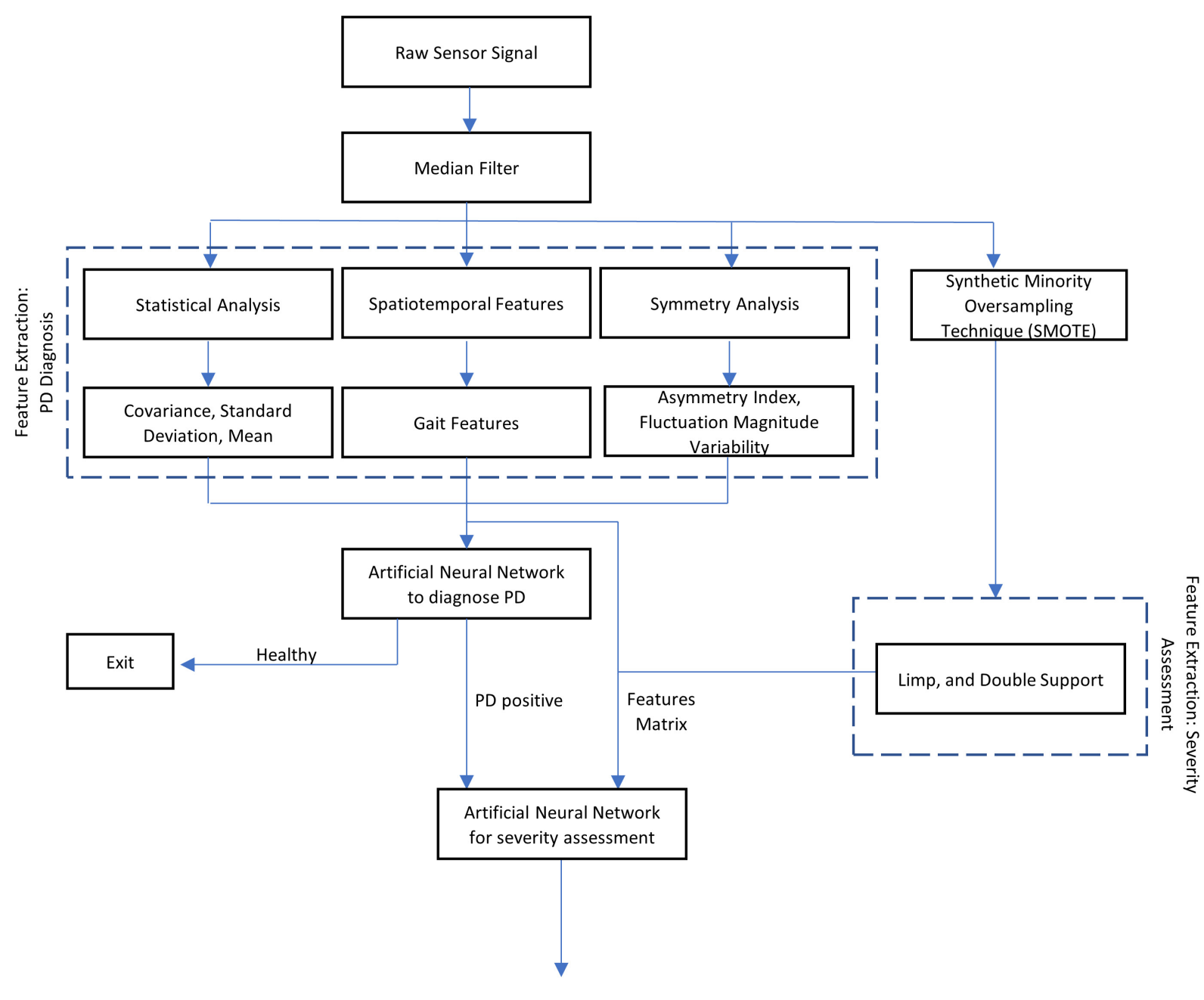

H\&Y Score

FIGURE 2 | Flowchart of the research methodology used in this study. 


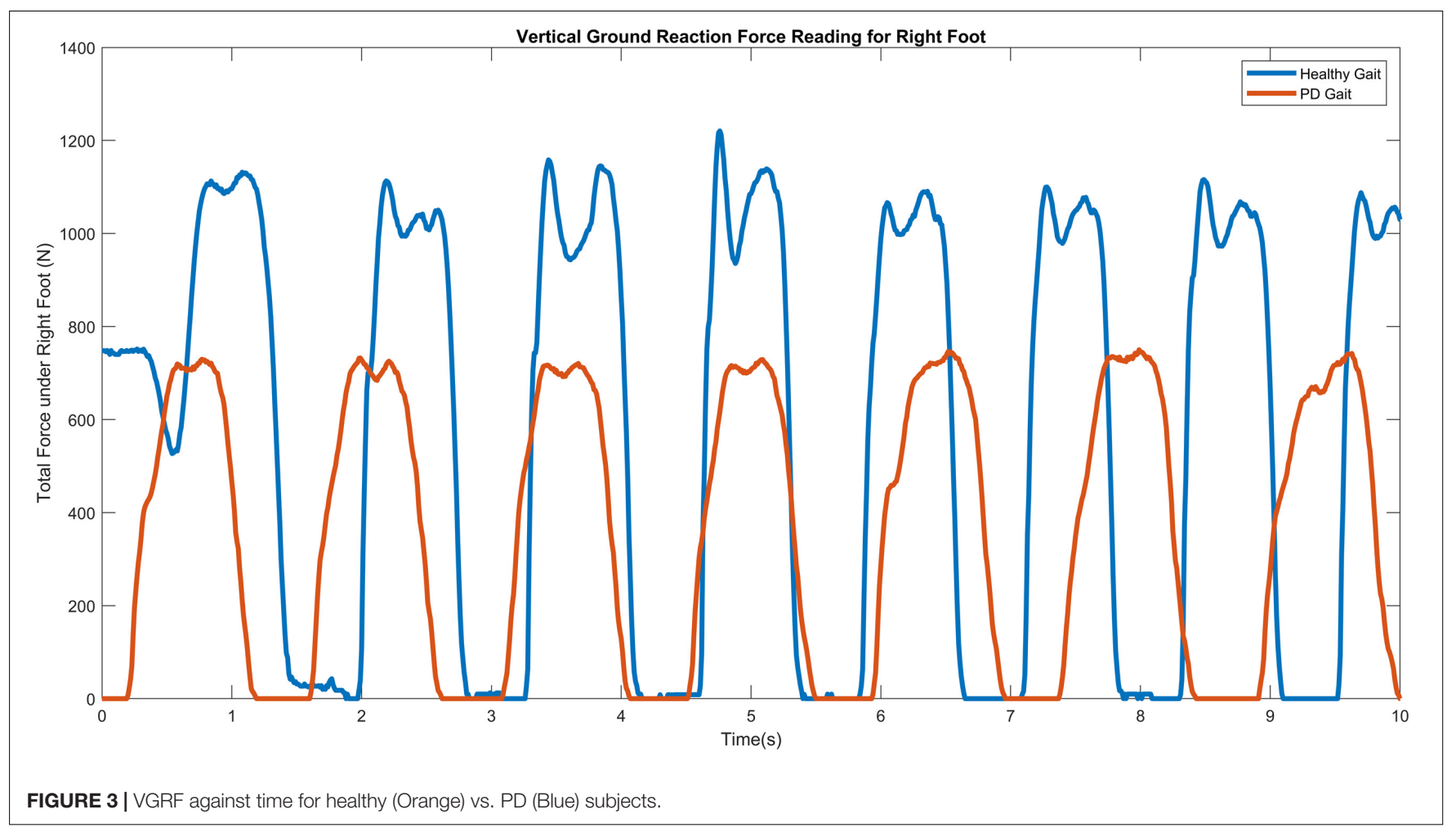

This filtered signal is then processed to retrieve useful gait features. The steps to process the data and extract useful features is illustrated in Figure 2.

\section{Feature Extraction for PD Diagnosis}

A PD subject generally records approximately $40 \%$ longer gait cycle time compared to a healthy subject, with notable lower stride velocity and stance periods, and as the disease progresses, patients may exhibit a single narrow peak force plot (Gaenslen and Daniela, 2010), characterized by a flat foot strike as opposed to a sharp heel strike in control subjects. Severe PD subjects may even exhibit toe-to-heel walking where the toe impacts the ground before the heel or mid-foot (Goetz et al., 2008). Figure 3 illustrates the difference in VGRF reading for a control vs. PD subject.

\section{Spatiotemporal features}

These features were extracted using the equations tabulated in Table 1, where $t$ represents the start and end indices of the gait cycle events, $\mathrm{T}$ represents time, $\mathrm{R}$ represents ratio, $\mathrm{V}$ represents velocity and $\mathrm{i}$ is the corresponding gait cycle iteration. To ensure accuracy of calculation, the summation of the swing and stance times were cross-checked against the stride time.

\section{Asymmetry indices}

Normalize the value of one side relative to the other, as shown in Eq. 8 which expresses the difference in the stance times of each foot as a fraction of the left stance time. This feature allows easy quantification of inter-individual comparisons (Nadeau, 2014). Using Eq. (8), the Asymmetry Index Ratio was calculated for each
TABLE 1 | Gait cycle time events.

\begin{tabular}{ll}
\hline Feature & Formula \\
\hline Gait cycle/ Stride time & $\mathrm{GCT}(\mathrm{i})=\mathrm{t}_{\text {start }}(\mathrm{i}+1)-\mathrm{t}_{\text {start }}(\mathrm{i})(1)$ \\
Stance time & $\mathrm{T}_{\text {stance }}(\mathrm{i})=\mathrm{t}_{\text {end }}(\mathrm{i})-\mathrm{t}_{\text {start }}(\mathrm{i})(2)$ \\
Swing time & $\mathrm{T}_{\text {swing }}(\mathrm{i})=\mathrm{t}_{\text {start }}(\mathrm{i}+1)-\mathrm{t}_{\text {end }}(\mathrm{i})(3)$ \\
Stance ratio & $\mathrm{R}_{\text {stance }}(\mathrm{i})=\mathrm{T}_{\text {stance }}(\mathrm{i}) / \mathrm{T}_{\text {stride }}(\mathrm{i})(4)$ \\
Swing ratio & $\mathrm{R}_{\text {swing }}(\mathrm{i})=\mathrm{T}_{\text {stride }}(\mathrm{i}) / \mathrm{T}_{\text {stance }}(\mathrm{i})(5)$ \\
Swing-stance ratio & $\mathrm{R}_{\text {swing-stance }}(\mathrm{i})=\mathrm{T}_{\text {swing }}(\mathrm{i}) / \mathrm{T}_{\text {stance }}(\mathrm{i})(6)$ \\
Stride length & $\mathrm{T}_{\text {stride }} \times \mathrm{V}_{\text {stride }}(7)$
\end{tabular}

TABLE 2 | Highest fluctuation magnitude variability (FMV) exhibited by sensors 3 and 7 .

\begin{tabular}{lcccccccc}
\hline Sensor & $\mathbf{1}$ & $\mathbf{2}$ & $\mathbf{3}$ & $\mathbf{4}$ & $\mathbf{5}$ & $\mathbf{6}$ & $\mathbf{7}$ & $\mathbf{8}$ \\
\hline FMV & 0.69 & 0.15 & 2.45 & 1.27 & 2.12 & 1.71 & 2.22 & 0.9
\end{tabular}

gait cycle and averaged over the subjects' entire walking duration of 2 min to get their individual Asymmetry Index ratio.

$$
\begin{gathered}
\text { Asymmetry Index Ratio }= \\
\left|L_{\text {stride }}(i)-R_{\text {stride }}(i)\right| / L_{\text {stride }}(i)
\end{gathered}
$$

\section{Statistical analysis}

Coefficient of variation (CV), Mean and Standard Deviation (STD) were chosen to assess gait variability in control and PD subjects. This is calculated for Left (L1 to L8) and Right (R1 to R8) feet for both PD and control subjects. Then, the variability in fluctuation magnitude can be computed for each of the eight 
TABLE 3 | Summary of unique extracted input features.

\begin{tabular}{|c|c|c|}
\hline Feature & $\mathbf{n}$ & Description \\
\hline Primary feature vector & 4 & $\begin{array}{l}\text { A vector consisting of: - Normalized Aggregated Left VGRF - Normalized Aggregated Right VGRF - FMV for sensor } 3 \text { - } \\
\text { FMV for sensor } 7\end{array}$ \\
\hline Stance times & 2 & Left and Right Stance Times \\
\hline Stride times & 2 & Left and Right Stride Times \\
\hline Swing times & 2 & Left and Right Swing Times \\
\hline Swing ratio & 2 & Left and Right Swing Ratios \\
\hline Swing ratio & 2 & Left and Right Stance Ratios \\
\hline Swing-stance ratio & 2 & Left and Right Swing-Stance Ratios \\
\hline Initial contact force & 2 & Right and Left Maximum Initial Contact Force \\
\hline Terminal contact force & 2 & Right and Left Maximum Terminal Contact Force \\
\hline Statistical covariance & 4 & $\begin{array}{l}\text { Covariance (CoV) of each of the Feature Vector Elements: - CoV(Normalized Aggregated Left VGRF) - CoV(Normalized } \\
\text { Aggregated Right VGRF) - CoV(FMV for sensor 3) - CoV(FMV for sensor } 7)\end{array}$ \\
\hline Statistical mean & 4 & $\begin{array}{l}\text { Mean of each of the Feature Vector Elements: - mean(Normalized Aggregated Left VGRF) - mean(Normalized } \\
\text { Aggregated Right VGRF) - mean(FMV for sensor 3) - mean(FMV for sensor } 7)\end{array}$ \\
\hline Standard deviation & 4 & $\begin{array}{l}\text { Mean of each of the Feature Vector Elements: - std(Normalized Aggregated Left VGRF) - std(Normalized Aggregated } \\
\text { Right VGRF) - std(FMV for sensor 3) - std(FMV for sensor 7) }\end{array}$ \\
\hline Step distance & 1 & Average Step Distance of the Subjects \\
\hline Asymmetry index & 1 & Asymmetry Index of the Stride between left and right feet \\
\hline Total Features & 34 & \\
\hline
\end{tabular}

sensors as shown in Figure $\mathbf{1}$ which is given by the difference in left and right sensor readings for each sensor as shown in Eq. 9.

Fluctuation Magnitude Variability $(F M V)=$

$$
|L i-R i| / \mathrm{Li} \quad \times 100 \%
$$

where $\mathrm{L}_{i}$ and $\mathrm{R}_{i}$ represent the Left or Right $i^{\text {th }}$ sensor, and $i$ is the sensor number corresponding to Figure 1. We determined that sensors number 3 and 7 demonstrate the highest variability, as shown in Table 2 and thus were selected as inputs to the classifier.

Table 3 summarizes the total list of feature vectors composed of the gait features and their statistical analyses to result in a total of 34 unique features as inputs to the classifier, where $n$ is the unique feature count.

\section{SMOTE and Feature Extraction for PD Severity Assessment}

During feature extraction, it was observed that the H\&Y stages of the patients were unevenly distributed in the dataset, where each stage corresponds to a class in the dataset. A majority of the samples belonged to H\&Y stage 2 (59.14\%) and stage $2.5(30.1 \%)$ and only $10.75 \%$ of the samples are stage 3 , which is a large imbalance of class sizes. Since a network trained on a dataset with imbalanced classes can face problems distinguishing between different classes (An et al., 2001), this problem was addressed by generating synthetic samples of the minority class, known as Synthetic Minority Oversampling Technique (SMOTE) (Chawla et al., 2002). Table 4 shows the dataset sample distribution before and after SMOTE was performed.

A peak detector was used on the output of Sensor 1 and Sensor 8 to obtain the initial contact (IC) and terminal contact (TC) magnitude and times, respectively. Subsequently, the initial double stance period and terminal double stance period was calculated using the formula established in Salarian et al. (2004).
TABLE 4 | Summary of dataset classes and their sample sizes before and after synthetic sample generation.

\begin{tabular}{lccc}
\hline & Original & SMOTE Samples & After SMOTE \\
\hline Stage 1: H\&Y 3 & $10(10.75 \%)$ & 20 & $30(21.27 \%)$ \\
Stage 2: H\&Y 2.5 & $28(30.1 \%)$ & 28 & $56(39.71 \%)$ \\
Stage 3: H\&Y 2 & $55(59.1 \%)$ & 0 & $55(39.00 \%)$ \\
\hline
\end{tabular}

A validation check is performed to ensure the gait parameters obtained corresponds to the correct gait event. We assume that the order of events in each gait cycle are as follows: Initial Contact of right foot $\left(\mathrm{IC}_{R}\right)$, Terminal contact of left foot $\left(\mathrm{TC}_{L}\right)$, Initial Contact of left foot $\left(\mathrm{IC}_{L}\right)$ and Terminal Contact of the right foot $\left(\mathrm{TC}_{R}\right)$, as illustrated in Figure 4.

Then, the validation equation used is (9).

$$
I C_{L}(j)<T C_{R}(j-1)<I C_{R}(j)<T C_{L}(j)
$$

where $j$ is the corresponding gait cycle iteration. Any cycle that does not meet this validation condition is excluded from analysis. Using the validated data, the following features are obtained (Eq. 10 to 13), where $j$ represents the gait cycle, as shown in Table 5.

\section{Neural Network Model for PD Diagnosis Network Architecture and Training Parameters}

A pattern recognition network was created using MATLAB r2017b to study the performance of the extracted gait parameters. The 34 unique input features are input into the pattern recognition network as the input layer, which consists of one hidden layer and a binary target (PD or Healthy) in the output layer. The input features are normalized to $[-1,1]$ using MATLAB Neural Network Toolbox's mapminmax preprocessing parameter to remove data range differences i.e., approximations between 


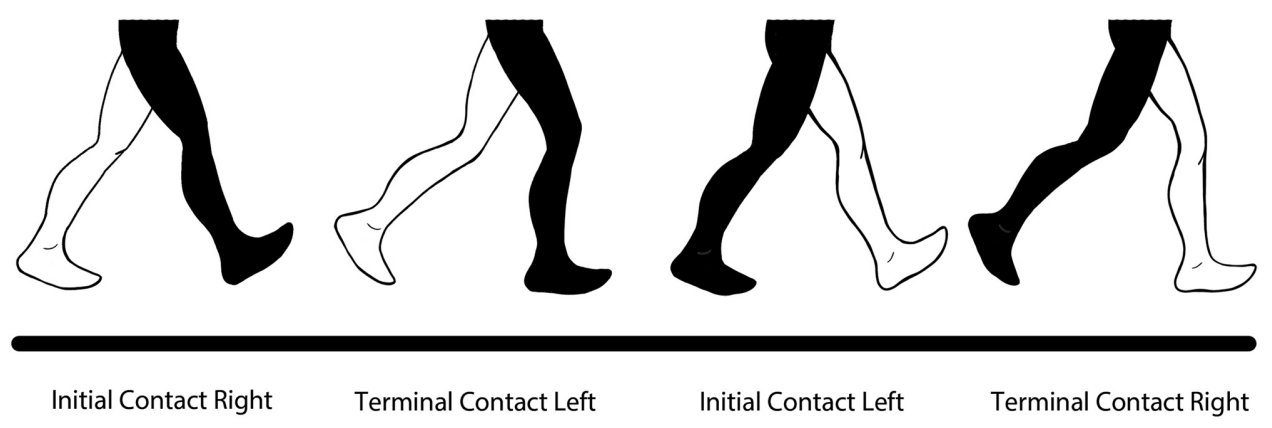

FIGURE 4 | Order of Gait Cycle Events used in the validation equation.

TABLE 5 | Spatiotemporal features extracted from VGRF data.

\begin{tabular}{lcc}
\hline \multicolumn{1}{l}{ Feature } & Formula & Eq. No. \\
\hline Initial Double Support & $I D S(j)=\frac{T C_{L}(j)-I C_{L}(j)}{G C T_{L}(j)} \times 100$ & $(10)$ \\
Terminal Double Support & $T D S(j)=\frac{T C_{R}(j-1)-I C_{R}(j)}{G C T_{L}(j)} \times 100$ \\
Double Support & $D S(j)=\mid D S(j)+T D S(j)$ \\
Limp & $L i m p(j)=|I D S(j)-T D S(j)|$ \\
\hline
\end{tabular}

large and small data values during training to reduce classification error. The architecture of the classifier was selected iteratively based on multiple sets of training algorithm and hidden unit patterns, out of which the best was selected. Figure 5 illustrates the different training algorithms tested for each hidden unit combination, where trainrp is resilient backpropagation, trainlm is Levenberg-Marquardt, traingdm is gradient descent with momentum, and trainscg is scaled conjugate gradient backpropagation. The best performance occurred for the resilient backpropagation algorithm (trainrp) with 25 hidden neurons and was therefore chosen as the network architecture.

\section{Neural Network Model for PD Severity Assessment}

An additional pattern classification network is used to classify the patients' disease progression based on spatiotemporal features into 3 separate classes for $\mathrm{H} \& \mathrm{Y}$ stages of 2, 2.5, and 3, respectively (as all the subjects in the database used fall under these three classes). The network architecture is selected based on iterative combinations of hidden units and training algorithms, which are shown in Figure 6. The tested algorithms include LevenbergMarquardt (trainlm), Scaled Conjugate Gradient (trainscg), Gradient Descent with Momentum (traingdm) and Resilient

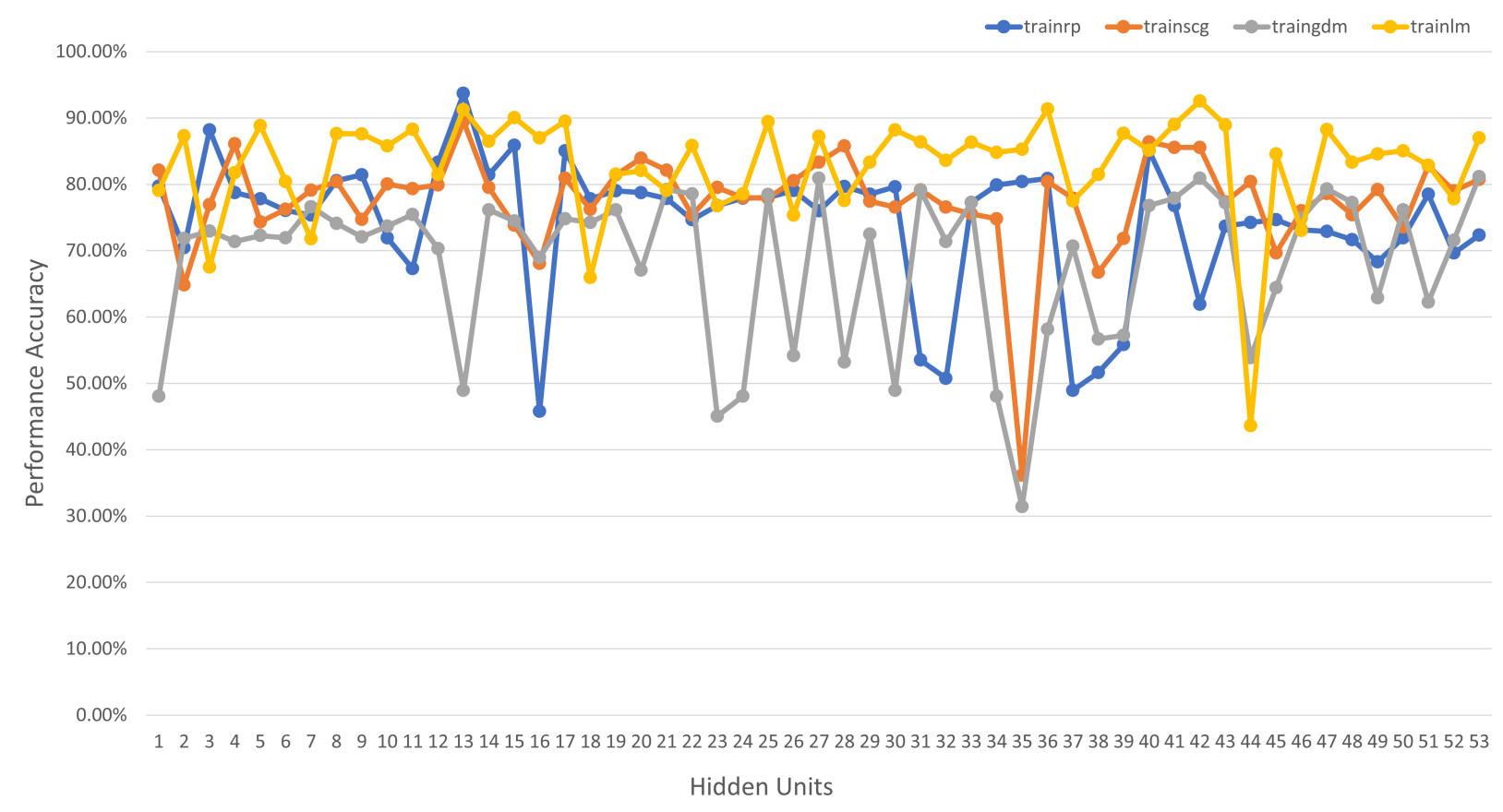

FIGURE 5 | Network performance vs. hidden unit count for different training algorithms for PD diagnosis. 


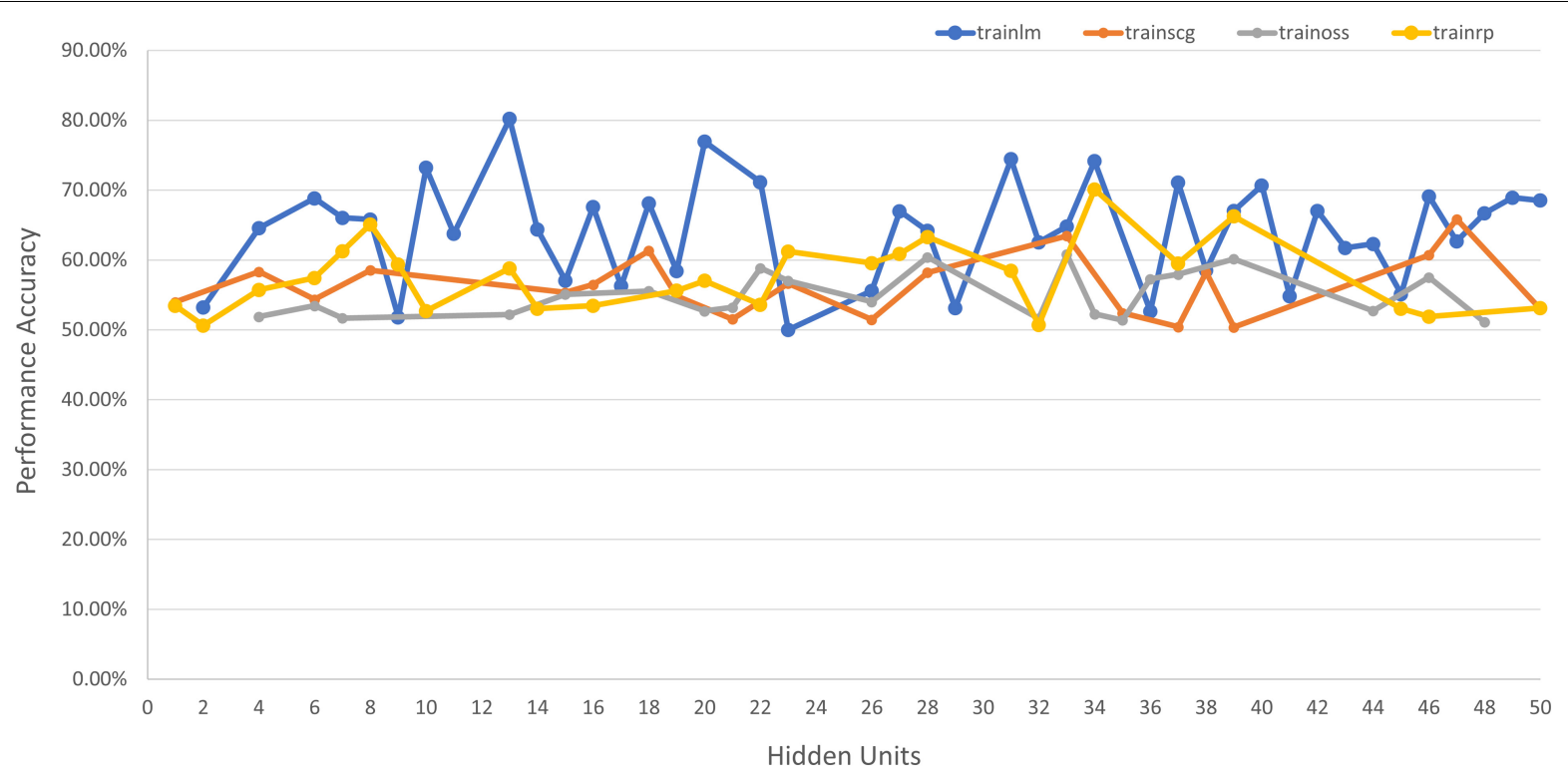

FIGURE 6 | Network performance vs. hidden unit count for different training algorithms for severity assessment.

Backpropagation (trainrp). The best performance was achieved using 13 neurons in the single hidden layer and the LevenbergMarquardt training algorithm (trainlm) and the hyperbolic tangent activation function.

\section{RESULTS AND DATA ANALYSIS}

\section{Performance Analysis for PD Diagnosis}

The pattern recognition network used for PD diagnosis performs well with a classification accuracy of $97.4 \%$ and a mean square error value of 0.0279 , which is consistent with literature that proves a high correlation between gait variability and presence of PD (Gaenslen and Daniela, 2010), thus resulting in an accurate classification. The accuracy (ACC), error (ERR), sensitivity (SN), specificity (SP), precision (PR) and false positive rate (FPR) also indicate good results, shown in Table 6.

Figure 7 shows the performance vs. loss for the chosen architecture for PD diagnosis, where the horizontal axis is the epoch count and the vertical axis depicts the Mean Squared Error (MSE) loss. It can be observed that after 62 epochs the network converges with an MSE of 0.079 on the validation and test data.

$\mathrm{K}$-fold cross validation and leave-one-out cross validation were used to ensure that the network performed as desired and did not overfit the data. Table 7 shows the results of cross validation performed and network performance. It can be observed that the model still performs well on different sets

TABLE 6 | The Accuracy (ACC), error (ERR), sensitivity (SN), specificity (SP), precision (PR) and false positive rate (FPR) for PD Diagnosis Classifier.

\begin{tabular}{cccccc}
\hline ACC & ERR & SN & SP & PREC & FPR \\
\hline 0.9741 & 0.0258 & 0.9770 & 0.9705 & 0.9770 & 0.0294
\end{tabular}

of unseen data, which shows good generalization ability and minimal overfitting (Cawley and Talbot, 2010).

\section{Performance Analysis for PD Severity Assessment}

Performance measures are usually represented as a confusion matrix for classification problems, where the rows and columns are the predicted and target class, respectively. The diagonals depict the correctly predicted samples (True Positive (TP) or True Negative (TN) and the off-diagonal cells correspond to the incorrectly classified samples (False Positive (FP) and False Negative (FN)). Additionally, the last column on the right shows the precision (positive predictive value) and the last row shows the recall rate (sensitivity or true positive rate) and the false negative rate. The cell on the bottom right depicts the overall accuracy (Mathworks, 2019). The confusion matrix for $\mathrm{H} \& \mathrm{Y}$ staging classifier is shown in Figure 8.

The H\&Y staging classifier performs well, with an accuracy of $87.1 \%$. It is also observed from the confusion matrix in Figure 8 that the H\&Y classifier has a precision of $90.5 \%$ and sensitivity of $67.9 \%$ for class 2 (H\&Y stage 2.5 ). This shows that the classifier is conservative for this class, but the opposite is true for class 3 (H\&Y stage 2) for which the classifier is biased (Santos et al., 2018). This may be attributed to the bias in the dataset, where more data is available for stage 2 and 2.5 compared to stage 3, which can skew results unfairly. Figure 9 shows the confusion matrix for the same network after implementing SMOTE techniques to balance the dataset.

It can be observed from Figure 9 that after SMOTE the classifier does not exhibit an unfair bias towards any particular class, while maintaining a similar prediction accuracy.

Using the additional data obtained from SMOTE techniques, we run multiple cross validation methods for testing and 


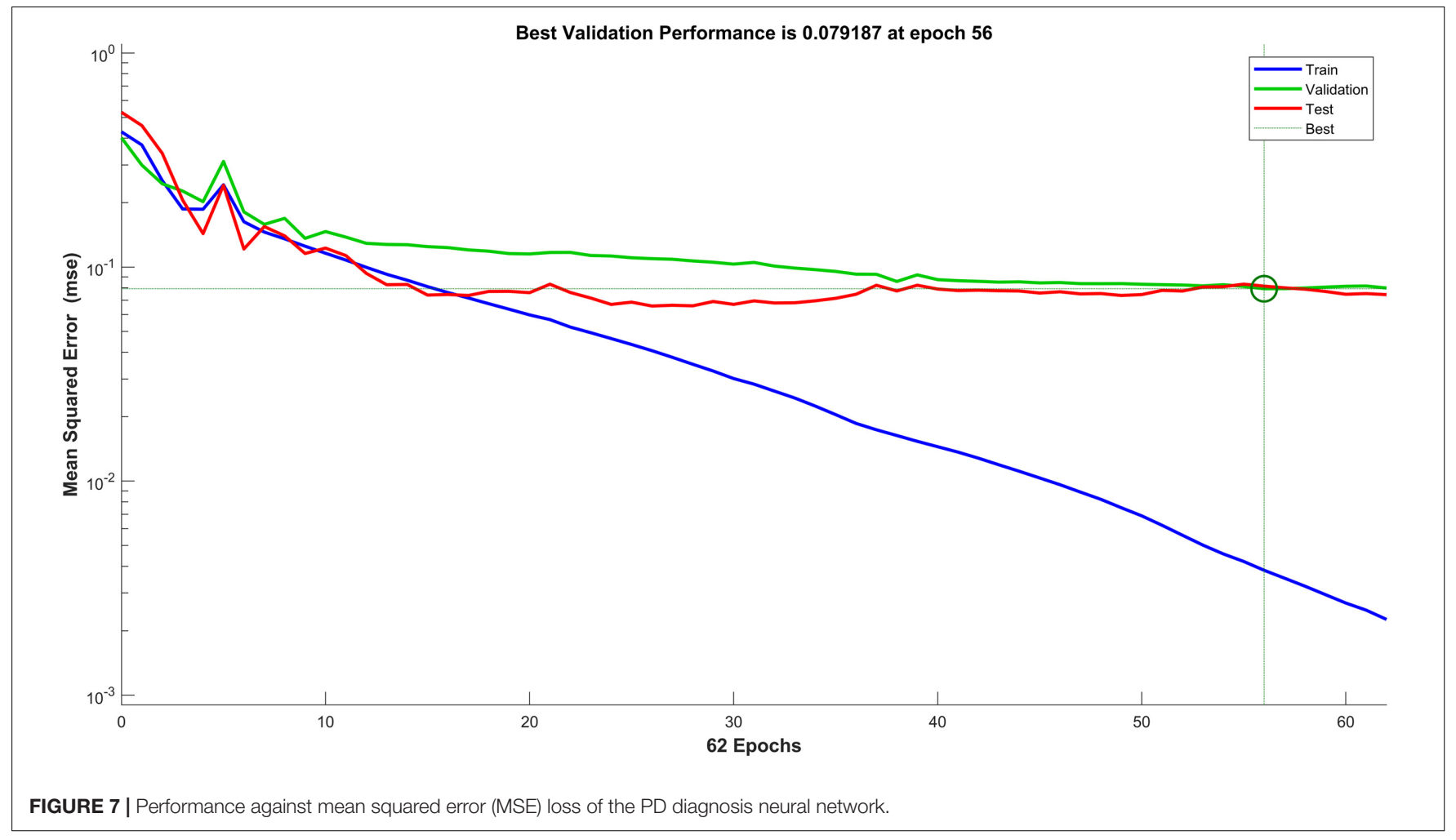

TABLE 7 | Results of different cross validation methods on the PD diagnosis classifier.

\begin{tabular}{lcc} 
Cross validation method (k) & MSE performance & Classification accuracy \\
\hline 1-fold & 0.0279 & $97.4 \%$ \\
2-fold & 0.1195 & $84.0 \%$ \\
5-fold & 0.0403 & $90.9 \%$ \\
10-fold & 0.0332 & $87.9 \%$ \\
Leave-one-out & 0.0287 & $94.9 \%$
\end{tabular}

error analysis, including $\mathrm{k}$-fold cross validation and leaveone-out cross validation. This helps against overfitting of the data. Table 8 shows the results of the cross validation performed for $\mathrm{PD}$ severity assessment and the respective network performance. It is observed that the network performs consistently over multiple $k$-values, which shows good generalization (Cawley and Talbot, 2010).

\section{DISCUSSION}

Good classification of PD subjects from healthy controls is achieved with an accuracy of $97.4 \%$ using input features extracted from VGRF data. Good accuracy of $87.1 \%$ was achieved in $\mathrm{H} \& \mathrm{Y}$ staging of patients' disease progression based on their spatiotemporal and kinetic features. However, as shown in Figure 8, the severity assessment data is unevenly distributed with majority of the samples being in H\&Y stage $2(59.14 \%)$ and stage $2.5(30.1 \%)$ and only $10.75 \%$ of the samples are

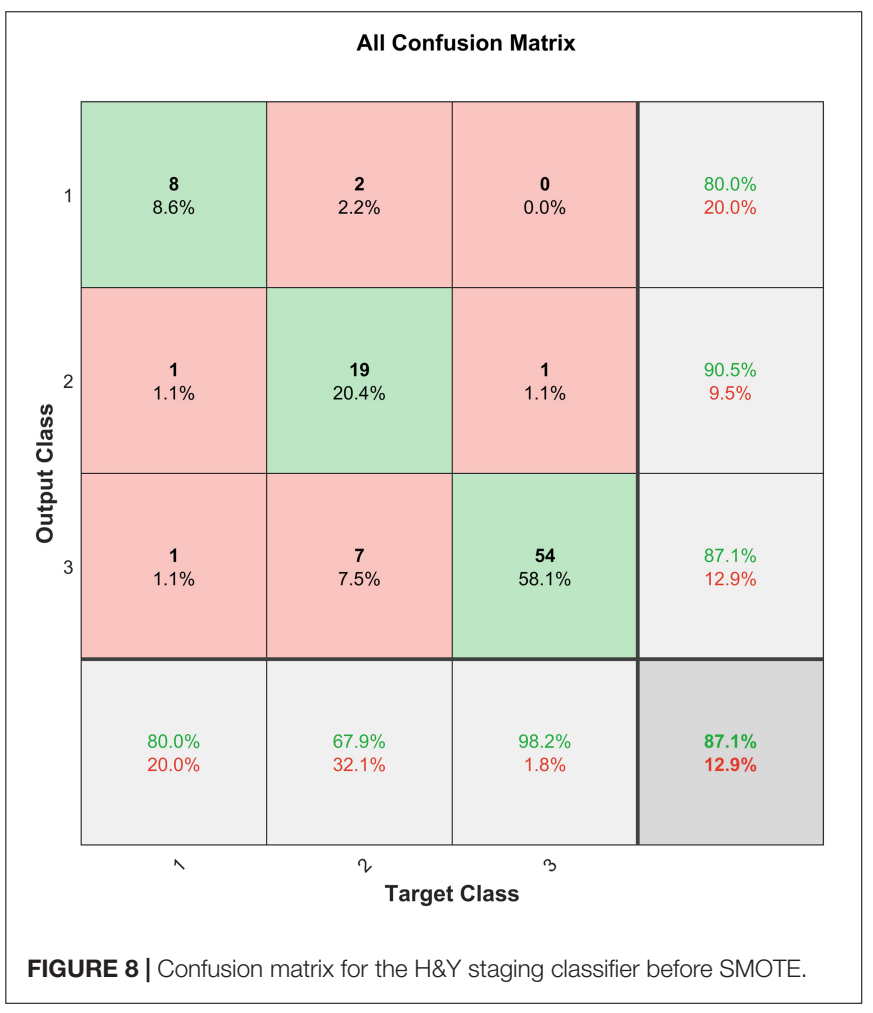

stage 3. Therefore, the classifier is biased towards the majority sample class, and this could affect the generalization ability of the classifier. This is dealt with using SMOTE (Chawla et al., 


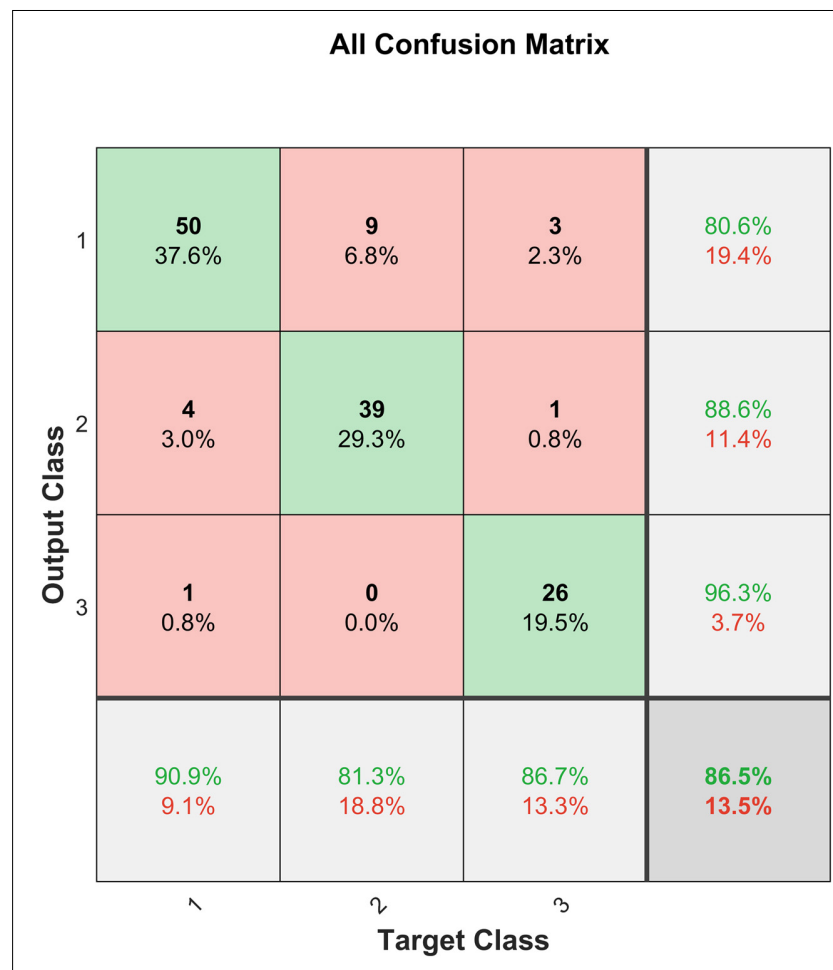

FIGURE 9 | Confusion matrix for the H\&Y staging classifier after SMOTE.

TABLE 8 | Results of different cross validation methods on the severity assessment classifier.

\begin{tabular}{lcc}
\hline Cross validation method (k) & MSE performance & Classification accuracy \\
\hline 1-fold & 0.0261 & $86.5 \%$ \\
5 -fold & 0.1063 & $73.06 \%$ \\
10-fold & 0.0905 & $76.08 \%$ \\
15-fold & 0.0873 & $77.3 \%$ \\
Leave-one-out & 0.0688 & $87.69 \%$ \\
\hline
\end{tabular}

2002), and the result is shown in Figure 9. It can be observed that the test accuracy improved due to the introduction of new samples in Class 1 (H\&Y Stage 3 ). This resulted due to the availability of more data samples for training, validation, and testing. Furthermore, as the dataset becomes more equally represented using SMOTE, the network was able to perform better on unseen data, with a test accuracy of $76.9 \%$, which is an improvement of $21.3 \%$. The network also exhibits lesser bias towards any particular class, and the overall accuracy is $87.2 \%$, which is also an improvement.

In comparison to the performance reported by (Manap et al., 2011; Lee and Lim, 2012; Perumal and Sankar, 2016; Zeng et al., 2016; Alam et al., 2017; Khoury et al., 2019), the proposed methodology in this work builds on and improves previous studies that use this VGRF database. This may be attributed to the extra analysis done to ensure the optimum network architecture was selected, and the combination of multiple features that proved successful in various past work, in addition to a new feature (Asymmetry Index) extracted using the same VGRF data.
The result achieved in this work also outperforms work that requires data to be collected via multiple sensors located at different parts of the patients' physique (Manap et al., 2011; Md Tahir and Manap, 2012; Klucken et al., 2013; Abdulhay et al., 2018). This is an added advantage for the proposed method, that it is able to prospectively diagnose PD with good accuracy using minimal data that may be obtained in a non-intrusive way via foot-worn sensors alone, for example embedded in subjects' shoes.

However, in 2018 (Aşuroğlu et al., 2018) proposed a Hybrid Machine Learning (ML) model (Locally Weighted Random Forest) and achieved a classification accuracy of 99\%. Though our classifier does not outperform this, it is worth noting that the work presented in this paper achieves a relatively close result using a comparatively less complicated network architecture. The classifier achieves an accuracy of $97.4 \%$ with a lightweight architecture and results that surpass or are competent with those achieved by complex methods such as Support Vector Machines (SVM) and Hybrid ML models.

Furthermore, the proposed method also carries out severity assessment corresponding to the H\&Y scale using features extracted VGRF data only, which is a scarcely researched area, as most researchers use additional information apart from VGRF, such as speech data to quantify disease progression (Salarian et al., 2004; Benmalek et al., 2015; Schlachetzki et al., 2017; Grover et al., 2018; Nilashi et al., 2018). However, there are studies that have performed better in terms of severity assessment using gyroscope and accelerometer data (Klucken et al., 2013) using complex models, but to the best of our knowledge, no studies use wearable sensor based VGRF data for this purpose. The proposed severity assessment method achieves a high accuracy in predicting patients' H\&Y scores using VGRF data. This was the expected outcome as spatiotemporal gait features show good correlation with H\&Y stages (Schlachetzki et al., 2017).

Furthermore, additional data generated from this study would also be useful in overcoming the bias exhibited by the classifier towards earlier H\&Y stages, as the current dataset is small and prone to overfitting, and exhibits a large imbalance in the distribution class samples.

We also successfully demonstrate the feasibility of the proposed novel approach of assessing PD severity using standalone VGRF data based on the H\&Y scale with an accuracy of $86.5 \%$ after SMOTE. Cross Validation methods also resulted in promising values of $76.08 \%$ for 10 -fold cross validation and $87.69 \%$ for leave-one-out cross validation. This shows that the classifier is able to generalize without overfitting or exhibiting bias towards any particular class.

Apart from its application in assisting clinicians in improving the accuracy of their assessments, this framework can also be implemented as a computational layer over smart wearables like smart insole shoes that can collect VGRF data, so that disease progression monitoring can be carried out remotely without requiring frequent clinic visits. This is possible as this is a lightweight ML architecture that does not require high processing power, thus making an integration with wearable sensors feasible. By reducing the frequency of clinical visits, this framework improves patients' and their caregiver's quality of life. 
As our framework operates on lightweight architecture and can be implemented online, it poses many benefits of portability, ease of use and functionality as opposed to non-portable gait analysis systems.

It is worth noting that this study is limited to the dataset size of control and PD subjects, and only investigates ground reaction forces in the vertical direction, as the dataset contains historical data that does not capture other directions of ground reaction forces. Although our study successfully showed that PD diagnosis and severity assessment can be done to a reasonable extent with VGRF only, further study is encouraged with a bigger sample size to investigate aspects such as predictive gait pattern tracking and integration with smart insole shoes to achieve a positive societal impact in the monitoring of movement disorders in the future.

\section{CONCLUSION}

A holistic, non-intrusive system is proposed for PD diagnosis and severity assessment using VGRF data from an online database collected from 166 subjects (93 PD and 73 healthy control subjects). A high classification accuracy of $97.4 \%$ is achieved using a simple ANN architecture, which confirms and extends the results of previous studies in this field that employ complex models to perform classification. Severity assessment is accurately carried out on the H\&Y scale to an accuracy of

\section{REFERENCES}

Abdulhay, E., Arunkumar, N., Narasimhan, K., Vellaiappan, E., and Venkatraman, V. (2018). Gait and tremor investigation using machine learning techniques for the diagnosis of Parkinson disease. Futur. Gener. Comput. Syst. 83, 366-373. doi: 10.1016/j.future.2018.02.009

Alam, M. N., Garg, A., Munia, T. T. K., Fazel-Rezai, R., and Tavakolian, K. (2017). Vertical ground reaction force marker for Parkinson's disease. PLoS One 12:175951. doi: 10.1371/journal.pone.0175951

An, A., Cercone, N., and Huang, X. (2001). "A case study for learning from imbalanced data sets," in Proceedings of the 14th Biennial Conference of the Canadian Society for Computational Studies of Intelligence, AI 2001: Advances in Artificial Intelligence, Ottawa, 1-15.

Aşuroğlu, T., Açıc1, K., Erdaş, ÇB., Toprak, M. K., Erdem, H., and Oğul, H. (2018). Parkinson's disease monitoring from gait analysis via foot-worn sensors. Biocybern. Biomed. Eng. 38, 760-772. doi: 10.1016/j.bbe.2018.06.002

Benmalek, E., Elmhamdi, J., Jilbab, A., and December, N. (2015). UPDRS tracking using linear regression and neural network for Parkinson's disease prediction. Int. J. Emerg. Trends Technol. Comput. Sci 4, 189-193.

Bhosale, T., Kudale, H., Kumthekar, V., Garude, S., and Dhumal, P. (2016). Gait analysis using wearable sensors. Int. Conf. Energy Syst. Appl. ICESA 2015, 267-269.

Cawley, G. C., and Talbot, N. L. C. (2010). On over-fitting in model selection and subsequent selection bias in performance evaluation. J. Mach. Learn. Res. 11, 2079-2107.

Chawla, N. V., Bowyer, K. W., Hall, L. O., and Kegelmeyer, W. P. (2002). SMOTE: synthetic minority over-sampling technique. J. Artif. Intell. Res. 16, 321-357. doi: 10.1613/jair.953

Gaenslen, A., and Daniela, B. (2010). Early diagnosis of Parkinson's disease. Int. Rev. Neurobiol. 90, 81-92.

Goetz, C. G., Tilley, B. C., Shaftman, S. R., Stebbins, G. T., Fahn, S., MartinezMartin, P., et al. (2008). Movement disorder society-sponsored revision of the unified Parkinson's Disease rating scale (MDS-UPDRS): scale presentation and clinimetric testing results. Mov. Disord. 23, 2129-2170. doi: 10.1002/mds.22340
87.1\% using features extracted only from VGRF data. The system as a whole is a simple and effective approach to PD diagnosis and severity assessment using only VGRF data obtained which is non-intrusive.

\section{DATA AVAILABILITY STATEMENT}

Publicly available datasets were analyzed in this study. This data can be found here: https://physionet.org/content/gaitpdb/1.0.0/.

\section{AUTHOR CONTRIBUTIONS}

SV and AG conceived and designed the study. SV analyzed the data. SV and AG drafted the manuscript. SV, AG, SA, and DG edited the manuscript. All authors read, commented, approved the final manuscript, contributed to the article, and approved the submitted version.

\section{FUNDING}

This work was supported by the Ministry of Higher Education, Malaysia under the project number FRGS/1/2016/TK04/MUSM/ 03/2 and the Advanced Engineering Platform of Monash University Malaysia.

Goldberger, A., Amaral, L., Glass, L., Hausdorff, J., Ivanov, P. C., Mark, R., et al. (2000). PhysioBank, physiotoolkit, and physionet: components of a new research resource for complex physiologic signals. Circulation [Online] 101, e215-e220.

Grover, S., Bhartia, S., Akshama, A. Y., and Seeja, K. R. (2018). Predicting severity of Parkinson's disease using deep learning. Proc. Comput. Sci. 132, 1788-1794. doi: 10.1016/j.procs.2018.05.154

Khoury, N., Attal, F., Amirat, Y., Oukhellou, L., and Mohammed, S. (2019). Datadriven based approach to Aid Parkinson's disease diagnosis. Sensors (Basel Swit.) 19:242. doi: 10.3390/s19020242

Klucken, J., Barth, J., Kugler, P., Schlachetzki, J., and Henze, T. (2013). Unbiased and mobile gait analysis detects motor impairment in Parkinson's Disease. PLoS One 8:e56956. doi: 10.1371/journal.pone.0056956

Koozekanani, S. H., Balmaseda, M. T. Jr., Fatehi, M. T., and Lowney, E. D. (1987). Ground reaction forces during ambulation in parkinsonism: pilot study. PubMed. Cent. 68, 28-30.

Lee, S. H., and Lim, J. S. (2012). Parkinson's disease classification using gait characteristics and wavelet-based feature extraction. Expert Syst. Appl 39, 73387344. doi: 10.1016/j.eswa.2012.01.084

Manap, H. H., Tahir, N. M. D., and Yassin, A. I. M. (2011). Statistical analysis of Parkinson disease gait classification using artificial neural network. IEEE Int. Symp. Signal Process. Inf. Technol. ISSPIT 2011, 60-65.

Mathworks (2019). DeepLearning Toolbox Documentation (R2019a), Mathworks. Available online at: https://www.mathworks.com/help/deeplearning/ref/ plotconfusion.html (accessed December 11, 2019).

Md Tahir, N., and Manap, H. H. (2012). Parkinson Disease gait classification based on machine learning approach.pdf. J. Appl. Sci. 12, 180-185. doi: 10.3923/jas. 2012.180.185

Nadeau, S. (2014). Understanding spatial and temporal gait asymmetries in individuals post stroke. Int. J. Phys. Med. Rehabil. 2:201.

Nilashi, M., Ibrahim, O., Ahmadi, H., Shahmoradi, L., and Farahmand, M. (2018). A hybrid intelligent system for the prediction of Parkinson's Disease progression using machine learning techniques. Biocybern. Biomed. Eng. 38, 1-15. doi: 10.1016/j.bbe.2017.09.002 
Parkinson's Foundation (2019). Understanding Parkinson's, 200 SE 1st Street, Ste 800, Miami, FL 33131, USA. Available online at: https: //www.parkinson.org/understanding-parkinsons (accessed November 23, 2019).

Perumal, S. V., and Sankar, R. (2016). Gait and tremor assessment for patients with Parkinson's disease using wearable sensors. ICT Express 2, 168-174. doi: 10.1016/j.icte.2016.10.005

Salarian, A., Russmann, H., Vingerhoets, F. J. G., Dehollain, C., Blanc, Y., Burkhard, P. R., et al. (2004). Gait assessment in Parkinson's Disease: toward an ambulatory system for long-term monitoring. IEEE Trans. Biomed. Eng. 51, 1434-1443. doi: 10.1109/tbme.2004. 827933

Santos, M. S., Soares, J. P., Abreu, P. H., Araujo, H., and Santos, J. (2018). Cross-validation for imbalanced datasets: Avoiding overoptimistic and overfitting approaches [Research Frontier]. IEEE Comput. Intell. Mag. 13, 59-76. doi: 10.1109/MCI.2018.286 6730

Schlachetzki, J. C. M., Barth, J., Marxreiter, F., Gossler, J., Kohl, Z., Reinfelder, S., et al. (2017). Wearable sensors objectively measure gait parameters in Parkinson's disease. PLoS One 12:e0183989. doi: 10.1371/journal.pone. 0183989
Stöckel, T., Jacksteit, R., Behrens, M., Skripitz, R., Bader, R., and Mau-Moeller, A. (2015). The mental representation of the human gait in young and older adults. Front. Psychol. 6:943.

Tadano, S., Takeda, R., and Miyagawa, H. (2013). Three dimensional gait analysis using wearable acceleration and gyro sensors based on quaternion calculations. Sensors (Switzerland) 13, 9321-9343. doi: 10.3390/s130709321

Zeng, W., Liu, F., Wang, Q., Wang, Y., Ma, L., and Zhang, Y. (2016). Parkinson's disease classification using gait analysis via deterministic learning. Neurosci. Lett. 633, 268-278. doi: 10.1016/j.neulet.2016.09.043

Conflict of Interest: The authors declare that the research was conducted in the absence of any commercial or financial relationships that could be construed as a potential conflict of interest.

Copyright (C) 2020 Veeraragavan, Gopalai, Gouwanda and Ahmad. This is an openaccess article distributed under the terms of the Creative Commons Attribution License (CC BY). The use, distribution or reproduction in other forums is permitted, provided the original author(s) and the copyright owner(s) are credited and that the original publication in this journal is cited, in accordance with accepted academic practice. No use, distribution or reproduction is permitted which does not comply with these terms. 